\title{
RAPIDLY-CONVERGING METHODS FOR SOLVING MULTILEVEL TRANSFER PROBLEMS
}

\author{
Eugene H. Avrett \\ Harvard-Smithsonian Center for Astrophysics \\ 60 Garden Street \\ Cambridge, MA 02138, USA \\ avrett@cfa.harvard.edu
}

\begin{abstract}
It is well known that lambda iterations can be used to solve multilevel non-LTE transfer equations in a reasonable number of iterations when the lambda operator is preconditioned, e.g., when the diagonal part of the operator is combined with other terms analytically. This approach is currently used successfully for the solution of model atoms with many line transitions, but sometimes a very large number of iterations is needed.

Lambda iteration consists of alternate solutions of the separate transfer and rate equations. For any given line transition the transfer and rate equations can be combined so that a solution can be obtained directly for that transition with no iterations needed between the transfer and rate equations. However, iterations are needed to determine the coupling between transitions. This can be time-consuming for model atoms with a large number of transitions that are treated in this way.

Here we show that 1) a hybrid approach involving such a direct solution for a few of the strongest transitions, and lambda iterations for the rest, gives rapid convergence, often with oscillations that need to be damped, and 2) this approach should include preconditioning of the lambda operator that occurs in the radiative coupling terms.

We illustrate these results with a simple three-level hydrogen atom and a finite, plane-parallel, symmetric atmosphere resembling a solar prominence, with a temperature of 8,000 $\mathrm{K}$ at the center, rising to very large values at each boundary (so that hydrogen is only partly ionized at the center and fully ionized at each boundary). Lambda iterations essentially fail to give a solution for this problem, while the hydrid solution converges in 5 to 10 iterations.
\end{abstract}

\section{INTRODUCTION}

The fundamental problem to be solved for low-density optically thick atmospheres where local thermodynamic equilibrium cannot be assumed is the simultaneous solution of the rate and transfer equations. The rate equations are used to determine the number densities of various energy levels at any location in the atmosphere given the angle-averaged radiation intensities at that location. The transfer equation is used to determine the radiation intensity at any location and direction given the number densities along a line extending from that location in the opposite direction. For simple problems the rate and transfer equations can be combined and solved directly, but this approach becomes impractical when the number of energy levels and radiative transitions is very large, because the coupling between transitions depends on the solution and must be treated iteratively.

Rather than solving the combined rate and transfer equations, one can iterate between the two. The transfer equation can be solved for the angle- and frequency-integrated mean intensity $\bar{J}$ at each depth given starting values of the number densities throughout the atmosphere. This $\bar{J}$ then can be 
used in the rate equations to obtain new number densities everywhere, replacing the starting values. These updated number densities can be used to obtain a new $\bar{J}$, etc. This is called the lambda iteration method. (The lambda operator is the function used to determine the mean intensity at one depth in terms of source-function values throughout the atmosphere.) Such a direct lambdaiteration method converges too slowly to be practical, but various techniques have been developed to achieve convergence in a reasonable number of iterations.

A comprehensive review of this topic has been provided by Hubeny (2003). The term "Accelerated Lambda Iteration" (ALI) ordinarily refers to removing the diagonal elements (and sometimes principal off-diagonal elements) from the lambda operator and combining these elements analytically with other terms. "Acceleration" thus refers to the faster convergence that results. Typically the convergence is still rather slow, and monotonic, so that purely numerical acceleration techniques can be applied as well.

Here we formulate the ALI method in an equivalent way but with slightly different terminology than used in Hubeny (2003) and in earlier papers. Instead of using the term "acceleration" we refer to the special treatment of diagonal elements as "preconditioning".

We use the simple case of a two-level atom to illustrate the much faster convergence that results from preconditioning, and compare with a direct solution that requires no iteration.

For a three-level case we show how to obtain a direct solution for each transition, and how to derive the coupling terms that relate each transition to the others. We use preconditioning in these coupling terms. Only the strongest transitions need to be solved directly in this way. Preconditioned lambda iterations can be used for weaker transitions. We call this a hybrid approach.

Finally, we show an example for which the ALI method essentially fails, while such a hybrid method converges in 5 to 10 iterations.

\section{THE RADIATIVE TRANSFER AND RATE EQUATIONS}

The rate equation for level $m$ of an $\mathcal{N}$-level atom with a continuum is

$$
\frac{\partial n_{m}}{\partial t}+\nabla \cdot\left(n_{m} V_{m}\right)=\sum_{\substack{\ell=1 \\ \neq m}}^{\mathcal{N}} n_{\ell} P_{\ell m}+n_{\kappa} P_{\kappa m}-n_{m}\left(\sum_{\substack{\ell=1 \\ \neq m}}^{\mathcal{N}} P_{m \ell}+P_{m \kappa}\right)
$$

where $n_{a}$ is the number density of level $a, P_{a b}$ is the transition rate from $a$ to $b$ per atom in level $a$, $\kappa$ refers to the next higher ionization stage, and $V_{m}$ is the mean flow velocity of atoms in level $m$.

The bound-bound rates are

$$
\begin{gathered}
P_{u \ell}=A_{u \ell}+B_{u \ell} \bar{J}_{u \ell}+C_{u \ell}, u>\ell \\
P_{\ell u}=B_{\ell u} \bar{J}_{u \ell}+C_{\ell u}, u>\ell
\end{gathered}
$$

where $A$ and $B$ are the Einstein coefficients, $C_{\ell u}$ amd $C_{u \ell}$ are the collisional excitation and deexcitation rates, and $\bar{J}$ is the integrated mean intensity for the $u \ell$ transition, which must be calculated from the transfer equation.

The transfer equation for the $u \ell$ line transition is

$$
\frac{d I_{\nu}}{d s}=-\frac{h \nu_{u \ell}}{4 \pi} \varphi_{\nu}\left[\left(n_{\ell} B_{\ell u}-n_{u} B_{u \ell}\right) I_{\nu}-n_{u} A_{u \ell}\right]-\kappa_{\nu}^{C} I_{\nu}+\epsilon_{\nu}^{C}
$$


where $s$ is geometrical depth in the direction of the intensity $I_{\nu}$. Here, for simplicity, we assume complete frequency redistribution (CRD, i.e., absorption and emission have the same uncorrelated dependence on frequency $\nu$, and have the common profile function $\varphi_{\nu}$ ). Using the well-known relationships between the Einstein coefficients, we can write

$$
\frac{d I_{\nu}}{d s}=-\kappa_{\nu}^{L}\left(I_{\nu}-S^{L}\right)-\kappa_{\nu}^{C}\left(I_{\nu}-S_{\nu}^{C}\right)
$$

where the line absorption coefficient is

$$
\kappa_{\nu}^{L}=\frac{h \nu_{u \ell}}{4 \pi} \varphi_{\nu}\left(n_{\ell} B_{\ell u}-n_{u} B_{u \ell}\right)
$$

and the line source function is

$$
S_{u \ell}^{L}=\frac{2 h \nu_{u \ell}^{3} / c^{2}}{\left(g_{u} n_{\ell} / g_{\ell} n_{u}\right)-1} .
$$

For simple illustrative purposes we consider the plane-parallel semi-infinite case for which $\kappa_{\nu}^{C} \ll \kappa_{\nu}^{L}$. Then the transfer equation reduces to

$$
\mu \frac{d I_{\nu}}{d \tau_{\nu}}=I_{\nu}-S^{L}
$$

where $d \tau_{\nu}=\kappa_{\nu}^{L} d z, d z=-\mu^{-1} d s$, and where $\mu$ is the cosine of the angle between the direction of $I_{\nu}$ (along $d s$ ) and the inward normal direction.

Solving for $I_{\nu}$ in terms of $S^{L}$, the mean intensity $J_{\nu}=\frac{1}{2} \int_{-1}^{+1} I_{\nu}(\mu) d \mu$ is given by

$$
J_{\nu}\left(\tau_{\nu}\right)=\frac{1}{2} \int_{0}^{\infty} \mathrm{E}_{1}\left(\left|t-\tau_{\nu}\right|\right) S^{L}(t) d t
$$

where $\mathrm{E}_{1}$ is the first exponential integral. The mean intensity can be expressed in the discrete form

$$
J_{i k}=\sum_{j} W_{i j k}^{\Lambda} S_{j}^{L}
$$

where $i$ and $j$ are depth indices and $k$ is the frequency index. Various quadrature representations can be used to determine the lambda-operator weighting coefficients $W_{i j k}^{\Lambda}$ which depend on the monochromatic optical depths $\tau_{i k}$.

The integrated mean intensity is

$$
\bar{J}=\int \varphi_{\nu} J_{\nu} d \nu, \quad \int \varphi_{\nu} d \nu=1
$$

so that

$$
\bar{J}=\sum_{j} W_{i j}^{\Lambda} S_{j}^{L}, \quad W_{i j}^{\Lambda}=\int \varphi_{\nu} W_{i j k}^{\Lambda} d \nu .
$$

The net rate coefficient $\rho_{u \ell}$ is defined by 


$$
n_{u} A_{u \ell} \rho_{u \ell}=n_{u}\left(A_{u \ell}+B_{u \ell} \bar{J}_{u \ell}\right)-n_{\ell} B_{\ell u} \bar{J}_{u \ell}
$$

so that

$$
\rho_{u \ell}=1-\frac{\bar{J}_{u \ell}}{S_{u \ell}^{L}}
$$

We can write $\bar{J}_{i}$ (i.e., $\bar{J}$ at depth $i$ for the $u \ell$ transition) either as

$$
\bar{J}_{i}=\sum_{j} W_{i j}^{\Lambda} S_{j}^{L}, \quad \text { or as } \quad \bar{J}_{i}=S_{i}+\sum_{j} W_{i j}^{\Lambda-1} S_{j}^{L},
$$

where $W_{i j}^{\Lambda-1}=W_{i j}^{\Lambda}-U_{i j}$ and $U_{i j}$ is the unit matrix. Then we obtain

$$
\rho_{i}=-\frac{1}{S_{i}^{L}} \sum_{j} W_{i j}^{\Lambda-1} S_{j}^{L}
$$

to be used in the rate equations in place of $\bar{J}_{u \ell}$.

In this derivation we have assumed that the line source function is frequency-independent, but the general case with partial frequency redistribution (PRD), with the continuum included (and using either plane-parallel or spherical geometry), can be treated by methods very similar to those discussed here. (See Avrett \& Loeser 1984.)

\section{PRECONDITIONING}

Let $W_{i j}^{\Lambda-1}=d_{i}+W_{i j}^{r}$ where $d_{i}=W_{i i}^{\Lambda-1}$ and where $W_{i j}^{r}$ is the same as $W_{i j}^{\Lambda-1}$ but with zero diagonal elements. Then

$$
\rho_{i}=-d_{i}-\frac{1}{S_{i}^{L}} \sum_{j} W_{i j}^{r} S_{j}^{L}
$$

From equation (7), the $S_{i}^{L}$ term in the denominator (for the $u \ell$ transition) can be written as

$$
S_{u \ell}^{L}=\left(\frac{n_{u}}{n_{\ell}}\right) q_{u \ell}
$$

where

$$
q_{u \ell}=\left(\frac{g_{\ell}}{g_{u}}\right) \frac{2 h \nu_{u \ell}^{3} / c^{2}}{1-\left(g_{\ell} n_{u}\right) /\left(g_{u} n_{\ell}\right)} .
$$

Note that $q_{u \ell}$ has only a secondary dependence on $n_{u} / n_{\ell}$ except when stimulated emission is important. Equation (17) for $\rho_{u \ell}$ at depth $i$ may then be written as

$$
n_{u} \rho_{u \ell}=-n_{u} d_{u \ell}-n_{\ell} \chi_{u \ell}
$$

where, at depth $i$,

$$
\chi_{i}=\frac{1}{q_{i}} \sum_{j} W_{i j}^{r} S_{j}^{L}
$$


Expressing $n_{u} \rho_{u \ell}$ in terms of $d_{u \ell}$ and $\chi_{u \ell}$ in the rate equation gives much better results than using $\rho_{u \ell}$ obtained directly from $S_{u \ell}^{L}$.

\section{THE SIMPLE TWO-LEVEL ATOM}

The time-independent rate equation for a two-level atom without a continuum, and without mass flow, is

$$
n_{2}\left(A_{21}+B_{21} \bar{J}_{21}+C_{21}\right)=n_{1}\left(B_{12} \bar{J}_{21}+C_{12}\right),
$$

or

$$
n_{2}\left(A_{21} \rho_{21}+C_{21}\right)=n_{1} C_{12} .
$$

Using $n_{2} \rho_{21}=-n_{2} d_{21}-n_{1} \chi_{21}$ gives

$$
n_{2}\left(-A_{21} d_{21}+C_{21}\right)=n_{1}\left(A_{21} \chi_{21}+C_{12}\right)
$$

or

$$
\frac{n_{2}}{n_{1}}=\frac{A_{21} \chi_{21}+C_{12}}{C_{21}-A_{21} d_{21}}
$$

Let $R=n_{2} / n_{1}$ and ignore stimulated emission. Then at depth $i$,

$$
R_{i}=\frac{\sum_{j} W_{i j}^{r} R_{j}+\epsilon_{i} R_{i}^{*}}{\epsilon_{i}-d_{i}}
$$

where $\epsilon=C_{21} / A_{21}$ and

$$
R_{i}^{*}=C_{12} / C_{21}=\left(\frac{g_{2}}{g_{1}}\right) \exp ^{-h \nu_{21} / k T}=n_{2}^{*} / n_{1}^{*} .
$$

This shows that $n_{2} / n_{1}$ approaches the LTE ratio $n_{2}^{*} / n_{1}^{*}$ at large optical depths, as $d_{i}$ and $W_{i j}^{r}$ approach zero.

Thus we can have three forms of the equation for $R_{i}$, as a result of:

1) preconditioning, using $W_{i j}^{r}=W_{i j}^{\Lambda-1}-d_{i}$ :

$$
R_{i}=\frac{\sum_{j} W_{i j}^{r} R_{j}+\epsilon_{i} R_{i}^{*}}{\epsilon_{i}-d_{i}}
$$

2) using $W_{i j}^{\Lambda-1}$ :

$$
R_{i}=\frac{\sum_{j} W_{i j}^{\Lambda-1} R_{j}+\epsilon_{i} R_{i}^{*}}{\epsilon_{i}}
$$

3) using the usual lambda operator, $W_{i j}^{\Lambda}$ :

$$
R_{i}=\frac{\sum_{j} W_{i j}^{\Lambda} R_{j}+\epsilon_{i} R_{i}^{*}}{1+\epsilon_{i}}
$$


Example 1:

Let $R_{i}^{*}=1$ and $\epsilon_{i}=0.01$ at all depths $i$ :

With preconditioning:

$$
R_{i}=\frac{\sum_{j} W_{i j}^{r} R_{j}+0.01}{0.01-d_{i}}
$$

Without preconditioning:

$$
R_{i}=\frac{\sum_{j} W_{i j}^{\Lambda} R_{j}+0.01}{1+0.01}
$$

Numerical solution:

$\begin{array}{llllllll}\tau & 0 & 0.1 & 1 & 3 & 10 & 30 & 100 \\ R & 0.1 & 0.123 & 0.260 & 0.476 & 0.842 & 0.999 & 1.000\end{array}$

Number of iterations required to reach $R=0.1 \pm 0.001$ at $\tau=0$

$\begin{array}{lcc} & \text { With preconditioning } & \text { Without preconditioning } \\ \text { initial } R=1 & 76 & 189 \\ \text { initial } R=0 & 79 & 334\end{array}$

Alternatively, as shown below, we can solve the set of linear equations for $R_{i}$ directly without any iterations, and in much less time than required by the preconditioned lambda iterations.

From

$$
R_{i}=\frac{\sum_{j} W_{i j}^{\Lambda-1} R_{j}+\epsilon_{i} R_{i}^{*}}{\epsilon_{i}}
$$

we write

$$
R_{i}-\frac{1}{\epsilon_{i}} \sum_{j} W_{i j}^{\Lambda-1} R_{j}=R_{i}^{*}
$$

Then, if $M_{i j}^{-1}$ is the inverse of

$$
M_{i j}=U_{i j}-\frac{1}{\epsilon_{i}} W_{i j}^{\Lambda-1}
$$

the solution is

$$
R_{i}=\sum_{j} M_{i j}^{-1} R_{i}^{*} .
$$

\section{A THREE-LEVEL ATOM}

We illustrate the basic properties of the general multilevel case by considering a three-level atom. The rate equations for levels 2 and 3 (again ignoring other stages of ionization for simplicity) are 


$$
n_{2}\left(A_{21} \rho_{21}+C_{21}+C_{23}\right)=n_{1} C_{12}+n_{3}\left(A_{32} \rho_{32}+C_{32}\right)
$$

and

$$
n_{3}\left(A_{31} \rho_{31}+C_{31}+A_{32} \rho_{32}+C_{32}\right)=n_{1} C_{13}+n_{2} C_{23} .
$$

These two equations can be solved for the two unknowns $\left(n_{2} / n_{1}\right)$ and $\left(n_{3} / n_{1}\right)$ if, from the transfer equations, we know the values of $\rho_{21}, \rho_{31}$, and $\rho_{32}$. Ordinary lambda iteration consists of successively solving these two rate equations and the three transfer equations.

Alternatively, we can write

$$
n_{u} \rho_{u \ell}=-n_{u} d_{u \ell}-n_{\ell} \chi_{u \ell}
$$

to get a better-conditioned set of equations. This is the basic preconditioning step, as in the two-level case. The preconditioned rate equations for levels 2 and 3 are

$$
n_{2}\left(C_{21}-A_{21} d_{21}+C_{23}+A_{32} \chi_{32}\right)=n_{1}\left(C_{12}+A_{21} \chi_{21}\right)+n_{3}\left(C_{32}-A_{32} d_{32}\right)
$$

and

$$
n_{3}\left(C_{31}-A_{31} d_{31}+C_{32}-A_{32} d_{32}\right)=n_{1}\left(C_{13}+A_{31} \chi_{31}\right)+n_{2}\left(C_{23}+A_{32} \chi_{32}\right) .
$$

We can iterate between the $\chi$ values and the rate equations, just as in the two-level case, with slow convergence using $W_{i j}^{\Lambda}$, but with much faster convergence using $W_{i j}^{r}$.

Direct numerical solutions for each transition can be used in the multilevel case, just as in the two-level case. The above equations can be written as

$$
\left(\frac{n_{2}}{n_{1}}\right)\left(x_{21}+y_{32}\right)-\left(\frac{n_{3}}{n_{1}}\right) x_{32}=y_{21},
$$

and

$$
-\left(\frac{n_{2}}{n_{1}}\right) y_{32}+\left(\frac{n_{3}}{n_{1}}\right)\left(x_{31}+x_{32}\right)=y_{31}
$$

where

$$
y_{u \ell}=C_{\ell u}+A_{u \ell} \chi_{u \ell},
$$

and

$$
x_{u \ell}=C_{u \ell}-A_{u \ell} d_{u \ell} .
$$

Then we can eliminate $n_{3} / n_{1}$ to obtain

$$
\left(\frac{n_{2}}{n_{1}}\right)\left(x_{21}+\bar{x}_{21}\right)=y_{21}+\bar{y}_{21} .
$$

Finally, the coefficients $\epsilon_{21}$ and $B_{21}^{S}$ in the expression

$$
S_{21}^{L}=\frac{\bar{J}_{21}+\epsilon_{21} B_{21}^{S}}{1+\epsilon_{21}}
$$

can be expressed in terms of the $x$ and $y$ coefficients. The results are

$$
\epsilon_{21}=\epsilon_{21}^{a}-\beta_{21} \epsilon_{21}^{b}, \quad \beta_{21}=e^{-h \nu / k T},
$$


and

$$
B_{21}^{S}=\alpha_{21} \beta_{21}\left(\epsilon_{21}^{b} / \epsilon_{21}\right), \quad \alpha_{21}=2 h \nu_{21}^{3} / c^{2}
$$

where

$$
\epsilon_{21}^{a}=\frac{1}{A_{21}}\left(C_{21}+\bar{x}_{21}\right), \quad \epsilon_{21}^{b}=\frac{g_{1}}{g_{2} \beta_{21} A_{21}}\left(C_{12}+\bar{y}_{21}\right) .
$$

Then, from equation (45), we can obtain $n_{2} / n_{1}$ at each depth $i$ from the solution of the set of equations

$$
S_{i}^{L}-\frac{1}{\epsilon_{i}} \sum_{j} W_{i j}^{\Lambda-1} S_{j}^{L}=B_{i}^{S} .
$$

Here $\epsilon$ and $B^{S}$ depend on $\chi_{31}$ and $\chi_{32}$, but not on $\chi_{21}$.

We can derive similar equations for $S_{31}^{L}$ that depend on $\chi_{21}$ and $\chi_{32}$, and for $S_{32}^{L}$ that depend on $\chi_{21}$ and $\chi_{31}$.

The direct approach applied to each of the transitions would consist of assuming initial values of $\chi_{21}, \chi_{31}$, and $\chi_{32}$, and solving each set of simultaneous equations to get $S_{21}^{L}, S_{31}^{L}$, and $S_{32}^{L}$, thus giving new iterative values of $\chi_{21}, \chi_{31}$, and $\chi_{32}$ from equation (21). (Equation 21 represents the solution of the transfer equation.)

Preconditioned lambda iteration in this case would consist of substituting the initial $\chi$ values into equations (40) and (41) to obtain $n_{2} / n_{1}$ and $n_{3} / n_{1}$ at each depth, and then using equation (21) to obtain new $\chi$ values. Each lambda iteration needs fewer computations than the direct approach, but many more iterations are required, and sometimes the lambda iterations do not converge.

It is not necessary to apply the direct approach to all transitions, only to the strongest ones that control the large-scale behavior of the solution. Thus in Example 2 below we use such a hybrid method, solving the simultaneous equations for $S_{21}^{L}$ and $S_{31}^{L}$ but using preconditioned lambda iteration for the 32 transition, i.e., the $\chi_{32}$ used in equations (40) and (41) is determined directly from $n_{3} / n_{2}$ rather than by solving the set of simultaneous equations for $S_{32}^{L}$.

The derivation given above for a three-level atom with no continuum can be extended without difficulty to cases with an arbitrary number of bound levels together with other stages of ionization.

\section{Example 2:}

Consider a 3-level hydrogen atom with a continuum (i.e., with the bound-free rates in equation 1 included), and a finite, symmetric atmosphere extending over the geometrical depth range $-700 \leq$ $s(\mathrm{~km}) \leq+700$, with the total hydrogen density $n_{\mathrm{H}}=10^{11} \mathrm{~cm}^{-3}$, constant with depth, and the temperature varying as $T=8000 \exp \left(s^{2} / 10^{5}\right)$, so that $T=8000 \mathrm{~K}$ at $s=0$, and $T=10^{6} \mathrm{~K}$ at $s= \pm 700$. In this case $n_{\mathrm{H}} \approx n_{\mathrm{e}} \approx n_{\mathrm{p}}$. Let $n_{1}$ represent the number density of hydrogen atoms in level 1 calculated from 1) the rate equations that include the bound-free transitions, and 2) the transfer equations for continuum radiation as well as line radiation.

Numerical solution: $n_{1}=2.4 \times 10^{4}$ at each surface

$$
n_{1}=5.6 \times 10^{6} \text { at the center }
$$

Total line-center optical thickness: $\tau_{21}=19, \tau_{31}=3.0, \tau_{32}=0.0027$

Note that in this example there are large temperature variations, but not large optical depths. 
Parameters of the (21)-line solution:

$\begin{array}{lll}\epsilon & \frac{\text { surface }}{1.4 \times 10^{-4}} & \frac{\text { center }}{1.4 \times 10^{-5}} \\ B & 1.9 & 8.3 \times 10^{-8} \\ S & 4.9 \times 10^{-7} & 1.3 \times 10^{-6}\end{array}$

Results for two methods of solution:

$\underline{\text { Case I }}$

Direct solutions for (21) and (31)

Preconditioned lambda iterations for (32).

$<0.1 \%$ convergence

after 8 iterations;

solution oscillating

with diminishing changes

computer time $10 \mathrm{~min}$. $\underline{\text { Case II }}$

Preconditioned lambda iterations

for (21), (31), and (32)

$\underline{\text { far from convergence }}$

after 50 iterations;

$n_{2}($ center $)=19100$ and slowly

decreasing toward the

correct value of 129

computer time $>50 \mathrm{~min}$.

\section{CONCLUSIONS}

1. We illustrate the well-known improvement in lambda-iteration solutions that results from preconditioning.

2. The strongest line transitions of a multilevel atom should be treated by solving the simultaneous equations corresponding to the combined rate and transfer equations, and not by lambda iteration.

3. Preconditioning should be used to determine the coupling between transitions that are needed in the simultaneous-equation solutions.

The methods described here are used in the Pandora atmosphere program (Avrett \& Loeser 2003). I am very grateful to Rudolf Loeser for his continued collaboration in this work.

\section{REFERENCES}

Avrett, E. H. \& Loeser, R. 1984, Line Transfer in Static and Expanding Spherical Atmospheres, in Methods in Radiative Transfer, ed. W. Kalkofen, Cambridge Univ. Press, 341-379.

Avrett, E. H. \& Loeser, R. 2003, Solar and Stellar Atmospheric Modeling Using the Pandora Computer Program, in Modelling of Stellar Atmospheres, IAU Symp. No. 210, ed. N. Piskunov, W. W. Weiss, \& D. F. Gray, ASP, Ann Arbor, A21.

Hubeny, I. 2003, Accelerated Lambda Iteration: An Overview, in Stellar Atmosphere Modeling, ed. I. Hubeny, D. Mihalas, \& K. Werner, ASP Conf. Series, 288, 17-30. 